\title{
Alignment Controlled Growth of Single-Walled Carbon Nanotubes on Quartz Substrates
}

\author{
Jianliang Xiao, ${ }^{\dagger}$ Simon Dunham, ${ }^{\ddagger}$ Ping Liu, ${ }^{\S}$ Yongwei Zhang, ${ }^{\S}, \|$ Coskun Kocabas, ${ }^{\ddagger}$ \\ Lionel Moh, ${ }^{\ddagger}$ Yonggang Huang, ${ }^{*, t, \perp}$ Keh-Chih Hwang, ${ }^{*}$ Chun Lu, ${ }^{\nabla}$ Wei Huang, ${ }^{\circ}$ \\ and John A. Rogers ${ }^{*, \neq}, \bullet$
}

Department of Mechanical Engineering, Northwestern University, Evanston, Illinois 60208, Department of Materials Science and Engineering, University of Illinois, Urbana, Illinois 61801, Institute of High Performance Computing, Singapore 117528, Singapore, Department of Materials Science and Engineering, National University of Singapore, Singapore 119260, Singapore, Department of Civil and Environmental Engineering, Northwestern University, Evanston, Illinois 60208, Department of Engineering Mechanics, Tsinghua University, Beijing 100084, China, King Abdullah University of Science and Technology, Thuwal, Saudi Arabia, Jiangsu Key Laboratory for Organic Electronics and Information Displays, Nanjing University of Posts and Telecommunications, Nanjing 210046, China, and Beckman Institute and Seitz Materials Research Laboratory, University of Illinois, Urbana, Illinois 61801

Received August 5, 2009; Revised Manuscript Received October 11, 2009

\begin{abstract}
Single-walled carbon nanotubes (SWNTs) possess extraordinary electrical properties, with many possible applications in electronics. Dense, horizonally aligned arrays of linearly configured SWNTs represent perhaps the most attractive and scalable way to implement this class of nanomaterial in practical systems. Recent work shows that templated growth of tubes on certain crystalline substrates yields arrays with the necessary levels of perfection, as demonstrated by the formation of devices and full systems on quartz. This paper examines advanced implementations of this process on crystalline quartz substrates with different orientations, to yield strategies for forming diverse, but welldefined horizontal configurations of SWNTs. Combined experimental and theoretical studies indicate that angle-dependent van der Waals interactions can account for nearly all aspects of alignment on quartz with $\mathrm{X}, \mathrm{Y}, \mathrm{Z}$, and ST cuts, as well as quartz with disordered surface layers. These findings provide important insights into methods for guided growth of SWNTs, and possibly other classes of nanomaterials, for applications in electronics, sensing, photodetection, light emission, and other areas.
\end{abstract}

The attractive charge transport properties of single-walled carbon nanotubes (SWNTs) make them excellent candidates for various applications in electronics, ranging from radio frequency (rf) analog devices, ${ }^{1-4}$ to flexible circuits, ${ }^{5}$ to heterogeneous integration of such systems with silicon

* Towhom correspondence should be addressed:y-huang@ northwestern.edu and jrogers@uiuc.edu.

Department of Mechanical Engineering, Northwestern University.

* Department of Materials Science and Engineering, University of Illinois.

$\S$ Institute of High Performance Computing.

"Department of Materials Science and Engineering, National University of Singapore.

${ }^{\perp}$ Department of Civil and Environmental Engineering, Northwestern University.

\# Department of Engineering Mechanics, Tsinghua University.

$\nabla$ King Abdullah University of Science and Technology.

${ }^{\circ}$ Nanjing University of Posts and Telecommunications.

- Beckman Institute and Seitz Materials Research Laboratory, University of Illinois. complementary oxide semiconductor (CMOS) chips. ${ }^{6}$ Large scale, horizontally aligned arrays of SWNTs represent ideal formats for integration, with the demonstrated ability to yield devices with performance approaching expectation based on the intrinsic properties of the constituent SWNTs. ${ }^{1,2}$ Many different methods have been reported to produce such arrays. Flow alignment is one of the most widely explored strategies, implemented either during deposition from solution via the action of liquid flows ${ }^{5}$ or during chemical vapor deposition (CVD) via gas flows. ${ }^{8,9}$ In the case of solution deposition, surface chemical modifications of the substrate can provide additional levels of control. ${ }^{10}$ Electric fields, applied in an alternating current mode during solution deposition ${ }^{11}$ or in a direct current mode during CVD growth ${ }^{12}$ can also yield alignment, due to dielectrophoretic and induced dipole interactions, respectively. Although each of these methods has promise, none in their current state of development offers 
the levels of perfection in alignment and linearity, at densities (i.e., numbers of tubes per unit area) and average lengths useful for most applications in electronics. More recent work $^{13,14}$ indicates that improvements can be achieved by using wetting interactions and interface assembly to yield high density (10-20 SWNTs/ $\mu \mathrm{m})$ arrays with good, although not perfect, degrees of alignment. The use of SWNTs in solution suspension could represent a disadvantage, however, due to possible contamination by residual surfactant and the potential for degradation of the tubes during the solubilization process. By contrast, growth of SWNTs by CVD on crystalline substrates such as sapphire and quartz can produce nearly ideal arrangements of pristine, low-defect tubes, particularly when used with strategically patterned catalyst particles. ${ }^{15-23}$ The best results have been reported on quartz, where levels of alignment can be controlled to better than $0.01^{\circ}$, with linear shapes to within a few nanometers over lengths of many micrometers, in tubes with average lengths of hundreds of micrometers (up to $\sim$ millimeters). ${ }^{4,16-23}$ Densities can also be high, with peak values approaching $100 \mathrm{SWNTs} / \mu \mathrm{m} .{ }^{17}$ Although this growth process has already been exploited to build high-performance $\mathrm{n}$ and $\mathrm{p}$ channel devices, ${ }^{3,15,20,21}$ and even fully integrated systems such as nanotube transistor radios and oscillators, ${ }^{1,2}$ the underlying mechanisms are poorly understood and the achieved alignment in the arrays involve orientation only along one direction. This paper presents a comprehensive, combined theoretical and experimental study of the alignment process on quartz and presents strategies to use quartz with different crystal orientations to achieve not only aligned linear arrays, but also more complex, but still well-defined, layouts. The results account for the key aspects, ranging from previously reported trends to additional effects revealed by new results reported here. This basic understanding is essential for further development of this approach to aligned arrays or even to other arrangements, not only on quartz but, potentially, on crystalline substrates of other materials.

Figure 1 provides a schematic illustration of the modeled system. We consider four cases - quartz with X ([21̄10]), Y ([0110]), Z ([0001]), and ST cut $^{17}$ orientations - to examine all of the effects of crystal orientation on alignment. $\alpha$-Quartz is used in this study, since the transition between $\alpha$ - and $\beta$-quartz is very fast and highly reversible at $573{ }^{\circ} \mathrm{C}^{24,25}$ and the experimental observations are all made at room temperature. Tridymite may also form at $870{ }^{\circ} \mathrm{C}$, since the SWNT growth temperature is $925{ }^{\circ} \mathrm{C}$. However, previous studies show that the transition from quartz to tridymite requires foreign oxides and the transition rate is very slow even when they are well mixed. ${ }^{26}$ Under the experimental conditions of SWNT growth as described in the methods section, no or only a very negligible amount of tridymite should form. In any case, calculations for $\alpha$ - and $\beta$-quartz give the same preferred alignment directions. See Supporting Information for details. In addition to proper atomic descriptions of the substrate, we treat the SWNTs explicitly with suitable chiral and diameter dependent configurations of the carbon atoms. Several experimental observations suggest the validity of important simplifying assumptions for the modeling. First,

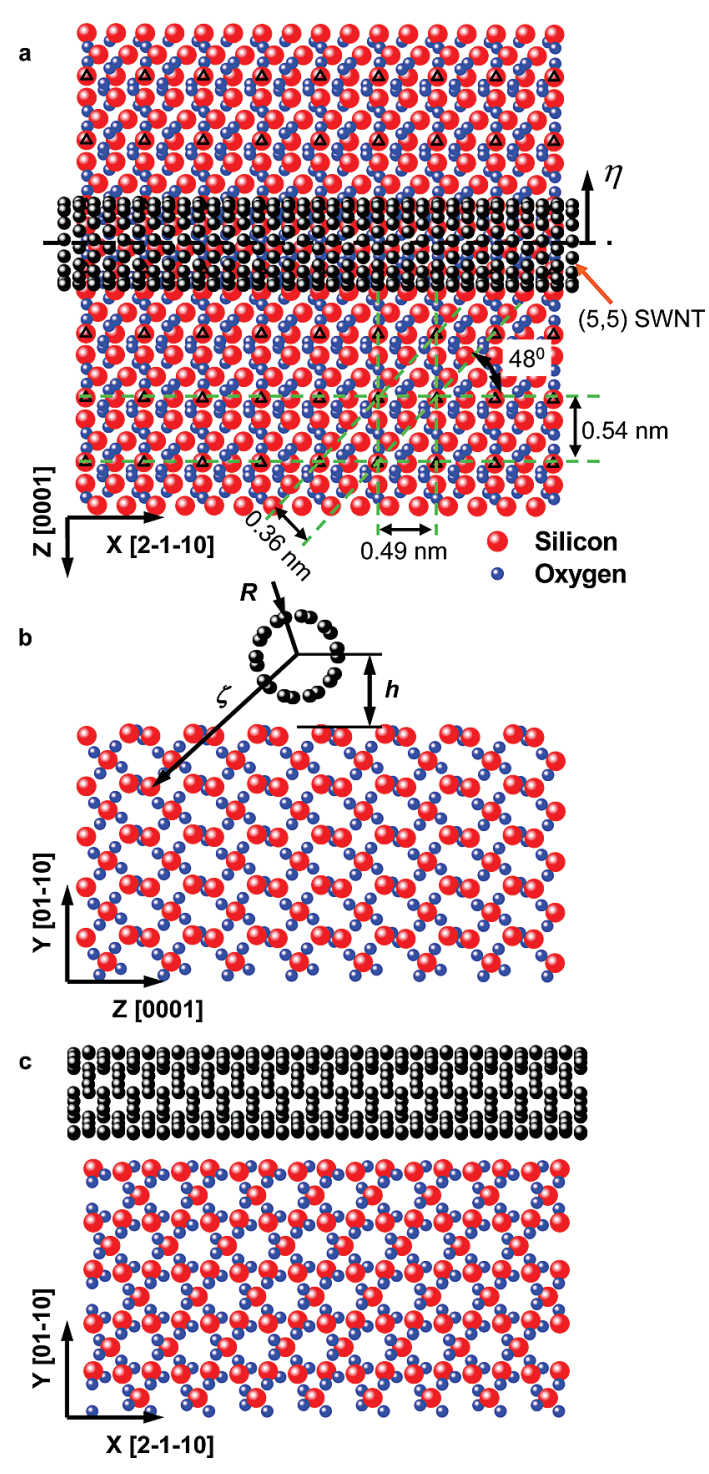

Figure 1. Schematic illustrations of a $(5,5)$ single-walled carbon nanotube (SWNT) resting on top of a Y cut quartz substrate: (a) top view, with key dimensions and variables identified; (b) end-on cross sectional view, with variables identified; (c) side cross sectional view.

in all cases, the SWNTs grown on Y or ST cut quartz show excellent degrees of alignment, uniformly and without any evident dependence on chirality or diameter, for values smaller than $\sim 1.5 \mathrm{~nm}$. This observation suggests that neither epitaxial registration with the substrate nor electronic properties of the SWNTs are critically important. Second, insensitivity of growth results to location or orientation of the quartz within the furnace or to spatial position across the substrate are consistent with uniform temperature distributions and, as a result, the absence of significant pyroelectric effects. Third, observations of alignment in SWNTs that fall back onto the substrate after growth in gas flows just above the substrate surface ${ }^{17,27,28}$ indicate that interactions between catalyst particles and the substrate can be neglected. Aligned SWNTs at spacings (minimum, as well as averages) that are smaller than the expected separations between adjacent step edges based on slight uncertainties in the cut angle ( $\mathrm{Y}$ cut) 

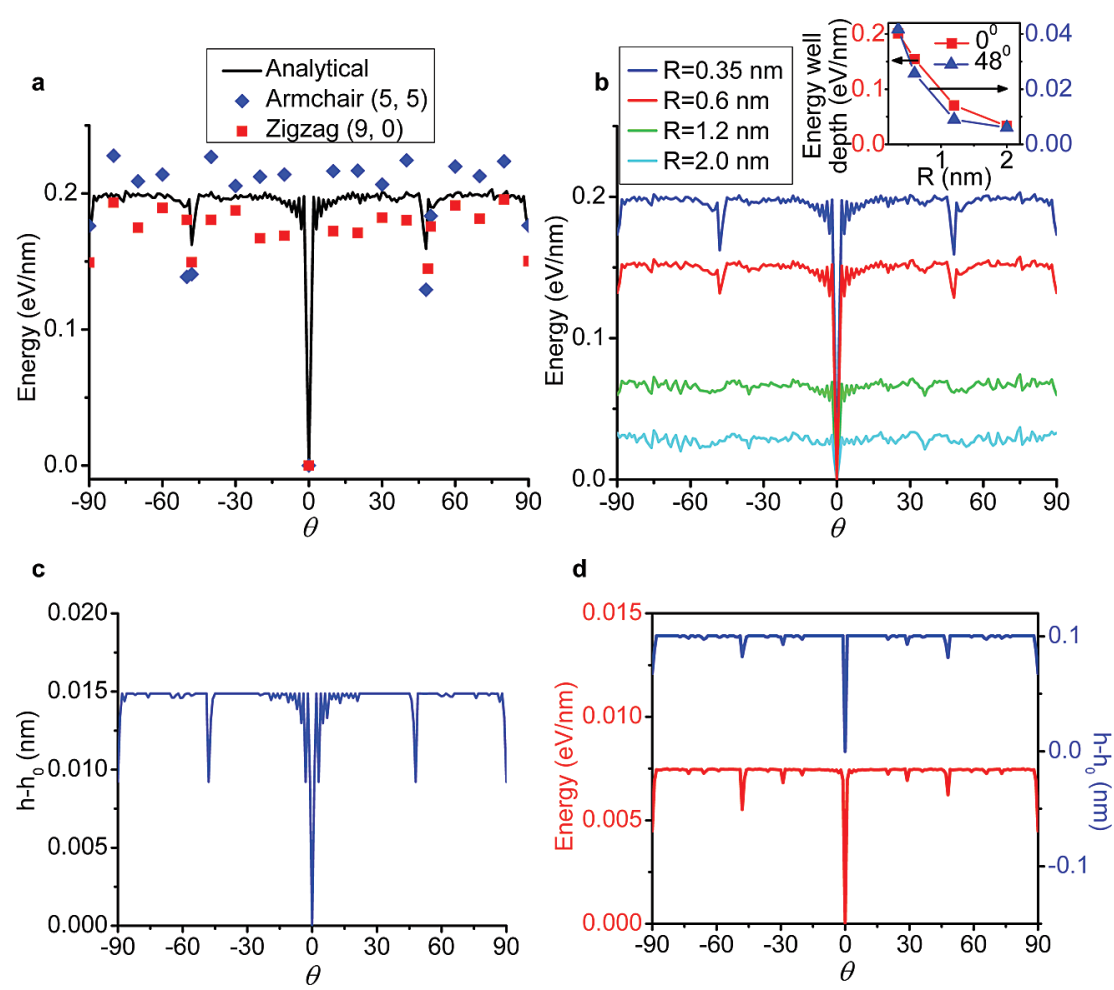

Figure 2. Orientation dependence of the van der Waals interaction energy between SWNTs and the surface of Y cut quartz. (a) Molecular modeling (symbols) and analytical calculations of interaction energies per unit length of SWNTs as a function of orientation angle $(\theta)$ on quartz. We refer to these plots as "energy maps". The good agreement between the results validates the analytical approach. Minima at $0^{\circ}$, $48^{\circ},-48^{\circ}$, and $90^{\circ}$ correspond to directions of preferred alignment. (b) Energy maps for the case of SWNTs with various radii $(R)$. The depths of the energy minima decrease with increasing $R$, as shown in the inset plot. (c) Change in height of a SWNT relative to the minimum height $\left(h_{0}\right)$ above the quartz $\left(h-h_{0}\right)$ as a function of orientation angle. The results show that the SWNT rests closer to the surface of the quartz in the preferred alignment directions, along $0^{\circ}, 48^{\circ},-48^{\circ}$, and $90^{\circ}$. (d) Interaction energy and $\left(h-h_{0}\right)$ as a function of angle for a SWNT $(R=0.6 \mathrm{~nm})$ on quartz, where the interactions are treated as hard spheres. The ability of this extremely simple model to capture the preferred alignment directions suggests an intuitive picture of the alignment process in which the SWNTs rest in molecular scale grooves on the surface of the quartz.

suggest that steps are unimportant. In addition, in certain experiments, SWNTs align in directions roughly perpendicular to clearly observable step edges, as described subsequently. Collectively, these observations suggest that it might be possible to treat the system with a simple model in which the alignment of SWNTs is governed by angledependent van der Waals ( $\mathrm{vdW}$ ) interactions, provided there is no significant permanent charge on the tubes or the quartz. (Polarization and piezeoelectric effects do not require net charge and can contribute to the interaction energy but they do not change the alignment preference, as discussed subsequently and in the Supporting Information.)

Using this view of the essential physics, we begin with molecular mechanics simulations based on empirical interatomic potentials ${ }^{29,30}$ to obtain the $\mathrm{vdW}$ interaction energies for $(5,5)$ armchair and $(9,0)$ zigzag SWNTs sitting on Y cut quartz, as shown in Figure 2a by blue and red dots, respectively. The orientation angle $\theta$ of the SWNT is measured from the $x$ direction ([2 $\overline{1} \overline{1} 0])$. The energy for $\theta=$ 0 is taken as zero as a reference state. The quartz surface is obtained by cutting between two oxygen layers, saturating the terminal oxygen atoms on the surface with hydrogen atoms and then reconstructing by ReaxFF (reactive force field) method, which can accurately simulate bond breaking and formation in silicon oxide systems. ${ }^{31}$ The obtained quartz surface structure is consistent with previous experimental ${ }^{32}$ and first principles dynamics studies ${ }^{33-36}$ (see Supporting Information for details). The SWNTs are sufficiently long such that the influence of local epitaxial registration is negligible. Figure 2a shows that both armchair and zigzag SWNTs have global energy minima at $0^{\circ}(x$ direction $)$, and local energy minima at $\pm 48^{\circ}$ and $\pm 90^{\circ}$ ( $z$ direction). The results indicate that SWNTs with similar diameter but different chiralities have negligible differences in their interaction energies with the substrate. Molecular dynamics simulations confirm that SWNTs aligned along the $0^{\circ}$ direction are stable at the growth temperature $(\sim 1200 \mathrm{~K})$.

These results suggest the applicability of a simple analytical model to represent the vdW interactions, in which the SWNT is treated as a continuous cylinder. These interactions can be represented by the Lennard-Jones potential, ${ }^{30}$

$$
V(r)=4 \varepsilon\left(\frac{\sigma^{12}}{r^{12}}-\frac{\sigma^{6}}{r^{6}}\right)
$$

where $r$ is the distance between two atoms, $\sigma=0.3621 \mathrm{~nm}$ and $\varepsilon=6.46 \times 10^{-3} \mathrm{eV}$ for $\mathrm{C}-\mathrm{Si}$ interaction, ${ }^{30,37,38} \sigma=$ $0.3340 \mathrm{~nm}$ and $\varepsilon=3.86 \times 10^{-3} \mathrm{eV}$ for $\mathrm{C}-\mathrm{O}$ interaction, ${ }^{30,37,38}$ and $\sigma=0.3110 \mathrm{~nm}$ and $\varepsilon=1.33 \times 10^{-3} \mathrm{eV}$ for $\mathrm{C}-\mathrm{H}$ interaction ${ }^{29,37}$ (see Supporting Information for details). For a long SWNT of radius $R$ sitting on top of the quartz 
surface, as shown in Figure 1, the vdW interaction between each atom in the substrate and the entire SWNT is the sum of $V(r)$ over all carbon atoms, which can be accurately represented by integration over the SWNT surface

$$
\phi=\int_{\text {SWNT surface }} V(r) \rho \mathrm{d} S
$$

where $\rho=38.2 \mathrm{~nm}^{-2}$ is the carbon atom density on the SWNT surface. ${ }^{40,41}$ For an atom in the substrate with a distance $\xi$ to the SWNT axis, the above integration is obtained analytically as

$$
\phi=4 \varepsilon \rho R\left(\sigma^{12} g_{12}-\sigma^{6} g_{6}\right)
$$

where

$$
\begin{array}{r}
g_{6}=\frac{\pi}{2(\zeta+R)^{3}(\zeta-R)^{4}}\left[4\left(\zeta^{2}+R^{2}\right) E\left(\frac{2 \sqrt{\zeta R}}{\zeta+R}\right)-(\zeta-R)^{2} K\right. \\
\left.\left(\frac{2 \sqrt{\zeta R}}{\zeta+R}\right)\right]
\end{array}
$$

and

$$
\begin{aligned}
g_{12} & =\frac{\left(\zeta^{2}+R^{2}\left(31 \zeta^{4}+194 \zeta^{2} R^{2}+31 R^{4}\right)\right.}{20\left(\zeta^{2}-R^{2}\right)^{6}} g_{6} \\
& -\frac{3 \pi\left(143 \zeta^{4}+482 \zeta^{2} R^{2}+143 R^{4}\right)}{320(\zeta+R)^{7}(\zeta-R)^{8}} E\left(\frac{2 \sqrt{\zeta R}}{\zeta+R}\right)
\end{aligned}
$$

and

$$
K(k)=\int_{0}^{\pi / 2} \frac{1}{\sqrt{1-k^{2} \sin ^{2} t}} \mathrm{~d} t
$$

and

$$
E(k)=\int_{0}^{\pi / 2} \sqrt{1-k^{2} \sin ^{2} t} \mathrm{~d} t
$$

are complete elliptic integrals of the first and second kind, respectively. The sum of $\phi$ for all the silicon, oxygen, and hydrogen atoms in the substrate gives the total van der Waals energy, which depends on the SWNT orientation $\theta$, distance $h$ between the SWNT axis and the substrate surface, and the shift $\eta$ along the substrate, normal to the long axis of the SWNT. For each given orientation $\theta$, minimization of the total energy with respect to $h$ and $\eta$ gives an "energy map", as shown in Figure 2a by a black curve for the Y cut case, where $\theta$ is measured from the $x$ direction, and the energy for $\theta=0$ is taken as zero, as for the molecular mechanics modeling. The long SWNT of Figure 2a has a radius of $0.35 \mathrm{~nm}$, close to that of $(5,5)$ armchair and $(9,0)$ zigzag SWNTs. The size of the quartz substrate was $20 \times$ $20 \mathrm{~nm}$; convergence was verified by comparing the results with substrates of size $40 \times 40 \mathrm{~nm}$. As shown in Figure 2a, the results given by this relatively simple analytical model agree well with the full molecular mechanics simulation, and predict a global energy minimum at $0^{\circ}$ ( $x$ direction), and local minima at $\pm 48^{\circ}$ and $\pm 90^{\circ}$ ( $z$ direction). Similar calculations performed on SWNTs with $R$ in the range typically observed from CVD deposition appear in Figure $2 \mathrm{~b}$, clearly showing energy minima at $0^{\circ}$ for SWNTs with $R$ equal to $0.35,0.6,1.2$, and $2 \mathrm{~nm}$. The depth of these energy wells decreases with increasing $R$.

The energetically favorable orientations, as illustrated by green dashed lines in Figure 1a, can be interpreted in terms of the atomic structure of the quartz. In Figure 1a, the top layer of silicon atoms, which are marked by black triangles, form a rectangular lattice. These silicon atoms also form parallel arrays when viewed along different directions. The $0^{\circ}$ ( $x$ direction) orientation gives the largest spacing between adjacent arrays of silicon atoms, $0.54 \mathrm{~nm}$ as shown in Figure 1a. This configuration allows the SWNTs to interact most effectively with quartz and hence gives the global energy minimum, consistent with a global minimum in $h$ at this same orientation. See Figure 2c. The local minima at $\pm 48^{\circ}$ and $\pm 90^{\circ}$ ( $z$ direction) can also be similarly explained since they correspond to the next two largest spacing between adjacent arrays of silicon atoms, 0.36 and $0.49 \mathrm{~nm}$ as shown in Figure 1a. Further insight into the underlying physics can be obtained by approximating the interaction between the tubes and the quartz as purely hard spheres (i.e., by taking limiting cases of the Lennard-Jones potential in which $\sigma$ for $\mathrm{C}-\mathrm{Si}$, $\mathrm{C}-\mathrm{O}$, and $\mathrm{C}-\mathrm{H}$ interactions are $1 / 10$ of the original values as given previously). Even this highly simplified analysis, shown in Figure 2d, captures the global and local minima in energy and $h$ along $0^{\circ}$. These results suggest that the surface interactions are dominant, and that one can intuitively consider the alignment as due to oriented "trenches" on the quartz with depths of $\sim 0.1 \mathrm{~nm}$. As $R$ increases, the significance of these trenches decreases, thereby reducing the depth of the energy well and the degree of alignment.

These trends are consistent with experimental observations of growth of SWNTs by CVD. (See the methods section for growth conditions.) Figure 3 shows some results. The SWNTs have a range of chiralities, with a diameter distribution illustrated by the histogram in the inset of Figure 3a. (This distribution is indistinguishable from that obtained in random networks of SWNTs grown on amorphous thermal oxides on silicon wafers using conditions and catalysts that are otherwise similar.) The primary alignment is along the $x$ direction, but on rare occasions $(\sim 0.1-4 \%$, depending on the sample) segments of SWNTs can be observed in welldefined off-axis orientations of $45 \pm 5^{\circ}$, as highlighted by circles in Figure $3 \mathrm{~b}$. The inset shows a histogram of the frequency of aligned segments versus angle, weighted by segment length, ignoring segments very close to the catalyst regions or those that show no strong alignment (most likely due to turbulent gas flow). In rare cases $(<0.1 \%)$ misaligned SWNTs at angles near $90^{\circ}$ were observed. We suspect that the rarity of SWNT segments at $90^{\circ}$ ( $z$ direction) is due to a shallower energy well compared to 0 or $\pm 48^{\circ}$. Furthermore, bending of a SWNT initially growing along $0^{\circ}$ toward $90^{\circ}$ requires higher energy compared to bending toward $48^{\circ}$. The orange arrow indicates the direction of gas flow. While it coincides with the alignment direction, the same characteristic behavior was observed for various flow directions. The same is true for all crystal orientations discussed subsequently indicating that gas flow does not play a major role in the observed alignment. Figure $3 \mathrm{c}$ shows the effect of diameter on alignment for $>60$ SWNTs measured by atomic force microscopy (AFM). For nanotubes less than $0.8 \mathrm{~nm}$ in diameter, almost no misalignment was observed over the 10 $\times 10 \mu \mathrm{m}$ scan they were observed over, while almost all nanotubes above $1.2 \mathrm{~nm}$ in diameter demonstrated some 


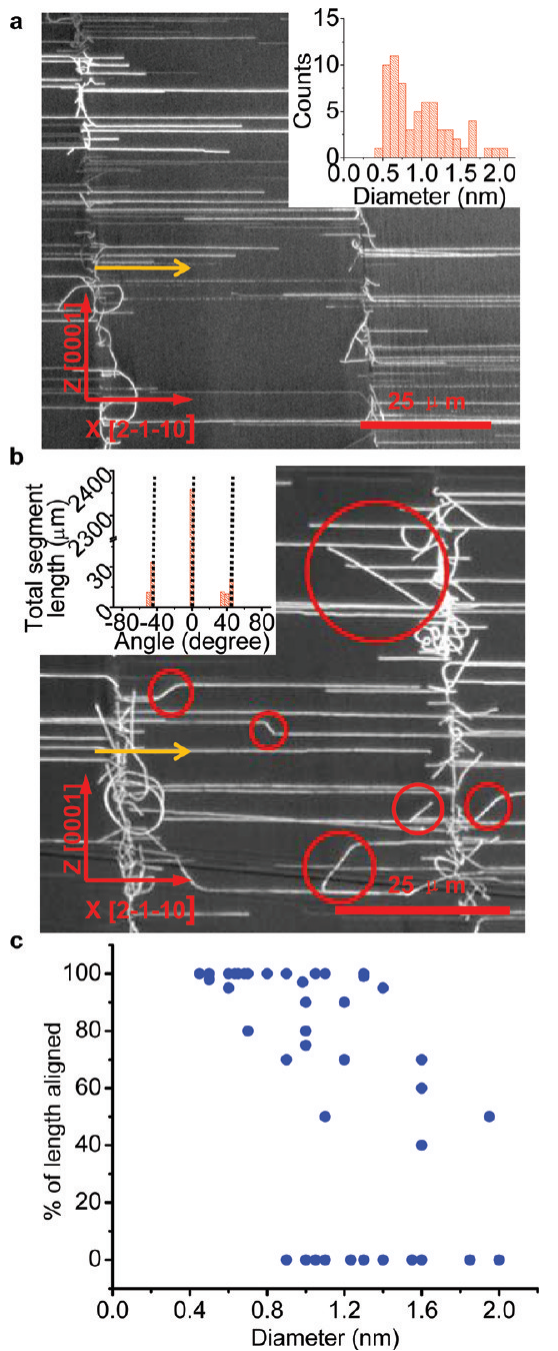

Figure 3. Scanning electron microscope (SEM) images of SWNTs grown by CVD on Y cut quartz substrates. (a) Large area SEM view of aligned SWNTs. Nearly all tubes align along the $x$ direction, independent of chirality or diameter, consistent with theoretical prediction. The inset provides the diameter distribution of the SWNTs shown here, as measured by AFM. (b) SEM image of aligned SWNTs on quartz, where short segments along the $45^{\circ}$ and $-45^{\circ}$ directions are visible. These directions correspond to local minima in the computed energy maps. The inset is a histogram showing the frequency of straight segments as a function of angle, weighted by segment length. Segments near the catalyst (within several micrometers) and winding SWNTs were ignored in this analysis. Both scale bars, $25 \mu \mathrm{m}$. The orange arrows indicate the flow direction. (c) Plot of the percentage of the lengths of SWNTs aligned at $0^{\circ}$ as a function of SWNT diameter. These results were generated from $10 \times 10 \mu \mathrm{m}$ AFM scans of $>100$ individual SWNTs.

degree of misalignment. In the intermediate diameter range, varying degrees of alignment were observed. All of these observations are consistent with the modeling results presented in Figure 2. Analysis that includes polarization and piezoelectric effects, hydroxylated, relaxed, and cleaved surfaces all show the same qualitative effects and the same preferred alignment directions. ${ }^{42-45}$ See Supporting Information for details.

Although this level of agreement is encouraging, one drawback of the model is that it assumes an atomically a

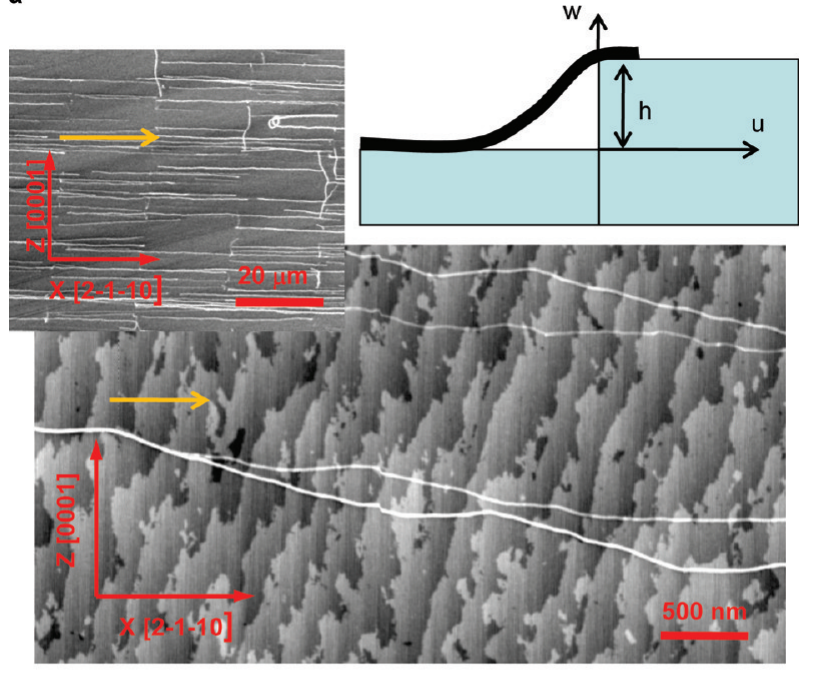

b

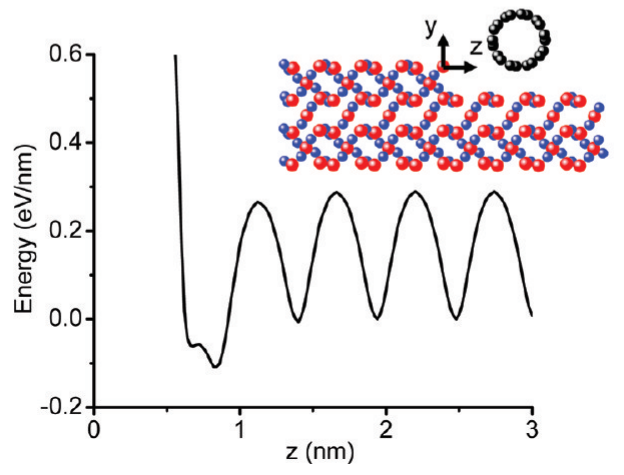

Figure 4. SEM and AFM images of SWNTs grown on a Y cut substrate that exhibits clear steps on its surface and the possible effects of those steps on alignment. (a) AFM (main; scale bar, 500 $\mathrm{nm}$ ) and SEM (upper left inset; scale bar, $20 \mu \mathrm{m}$ ) images of experimental results. The illustration in the upper right inset shows the crossing of a step by a SWNT. In this case, the preferred alignment direction ( $x$ direction) is roughly orthogonal to the directions of the step edges. This observation suggests that alignment can occur in directions that do not lie along step edges and that the growing SWNTs can climb over nanometer scale features of relief, consistent with mechanics modeling. The orange arrow indicates the flow direction. (b) Interaction energy as a function of distance between a step and the axis of the SWNT oriented along the direction of the step. The inset provides a schematic illustration. The minimum in energy near the step is slightly lower than the minima away from the step, but only by a modest amount. These results and corresponding ones based on molecular modeling suggest that step edges can aid in alignment but that they are not necessary for the alignment process.

smooth surface, whereas the actual substrates have root mean squared roughness in the range of $0.2-0.4 \mathrm{~nm}$ as measured by AFM with conventional tips, and in some cases step edges can be observed. (See Supporting Information.) Effects of finite roughness can be examined theoretically by considering the case of a SWNT that encounters a feature of relief as it is growing on the surface. The tube can either climb over this feature or turn to grow along it, depending on which gives the lower energy. ${ }^{46}$ The critical height of a feature (Figure 4a) below which a SWNT of radius $R$ can climb over can be shown to be 


$$
h<\frac{3 \pi(\pi-2)}{128} \sqrt{\frac{E t \pi R^{3}}{2 \gamma_{0}}}
$$

where $E t=0.42 \mathrm{TPa} \cdot \mathrm{nm}$ is the tension rigidity of SWNT, ${ }^{47,48}$ $\gamma_{0}$ is the adhesion energy between SWNT and quartz, and $\gamma_{0}=1.79 \mathrm{eV} / \mathrm{nm}$ for $R=0.6 \mathrm{~nm}$. See Supporting Information for details. This result, which is based purely on mechanics and ignores potentially important effects associated with the growth process, suggests that a SWNT of radius $0.6 \mathrm{~nm}$ can climb over any feature with height less than $1.88 \mathrm{~nm}$. According to this analysis, surface roughness of standard quartz wafers used here should not impede aligned growth. To demonstrate explicitly the relative insensitivity to moderate surface roughness and to provide information on the possible role of step edges, we grew tubes on $\mathrm{Y}$ cut wafers with a miscut of $\sim 0.1^{\circ}$, to create organized steps with relief heights of $\sim 0.4 \mathrm{~nm}$ oriented approximately about $20^{\circ}$ from the $z$-direction $\left(\theta=70^{\circ}\right)$. Experimentally, we observed the expected tube alignment, even though the direction is almost orthogonal to the step edges, as shown in Figure 4a. The overall alignment is somewhat worse than that on smoother, polished substrates used for the results of Figure 2. Previous publications indicate an ability to grow over sharp edges of relief with heights of $\sim 1-2 \mathrm{~nm}$, but not much more. ${ }^{17,18}$ Additionally, random surface roughness can contribute to lower degrees of alignment, although such systems are less well amenable to modeling due to the range of lateral length scales involved in the relief. Substrates polished to create varying degrees of surface roughness show very strong alignment for substrate with root-mean-square roughness up to $0.8 \mathrm{~nm}$ and show good alignment for even rougher surfaces.

These observations clearly demonstrate that aligned tubes can form without and, in fact, despite the presence of steps. They do not, however, rule out the possibility that steps could give rise to alignment in certain situations. Additional analysis can provide some insights. Figure $4 \mathrm{~b}$ shows the vdW energy of a SWNT of radius $0.35 \mathrm{~nm}$ adjacent to a step edge of height $0.43 \mathrm{~nm}$ for Y cut quartz. The SWNT and the step edge are along the $x$ direction, the preferred growth direction, and $z=0$ denotes the SWNT axis being on top of the step edge. The step edge clearly reduces the energy minimum, which is $0.11 \mathrm{eV} / \mathrm{nm}$ lower than the minimal energy away from the edge. This effect is, however, localized: the energy minimum occurs at $0.82 \mathrm{~nm}$ from the step edge, but drops to negligible values at distances of more than $\sim 1.1 \mathrm{~nm}$. These results agree with molecular dynamics simulations at 1200 K (see movies in Supporting Information). A $(5,5)$ armchair SWNT that is initially $1.0 \mathrm{~nm}$ from the step edge but forms a $5^{\circ}$ angle moves toward and becomes parallel to the step. For the same angle but at an average distance of $1.8 \mathrm{~nm}$, the SWNT aligns along the $x$ direction but does not move toward the step. For a typical commercially available Y cut substrate, the miscut angle is $<0.25^{\circ}$. This angle corresponds to an average separation between steps of $\sim 100 \mathrm{~nm}$, assuming step heights of $\sim 0.4 \mathrm{~nm}$. This distance is much larger than separations that are often observed between adjacent SWNTs in arrays grown on $\mathrm{Y}$ cut substrates. Collectively, these results rule out step edges as a major cause of alignment.

Although these observations and calculations indicate some insensitivity to surface topology due to steps or to random a

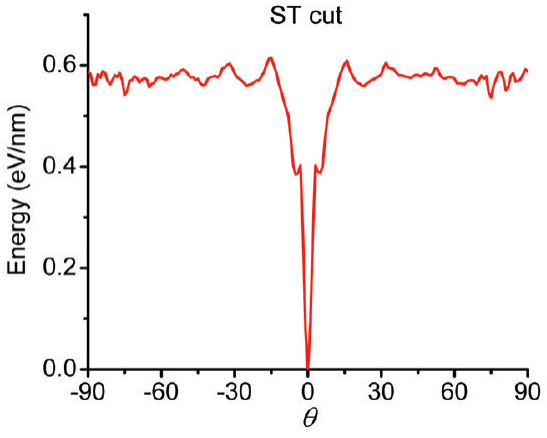

b

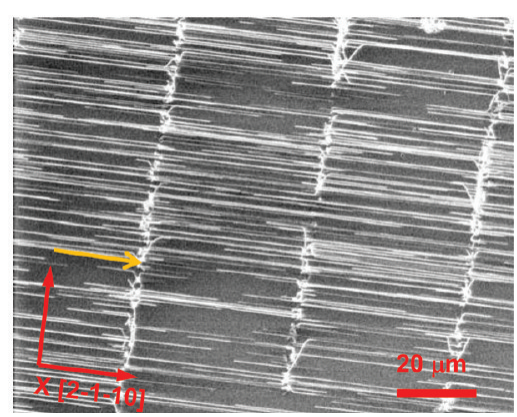

Figure 5. Interaction energy between a SWNT and ST cut quartz, as a function of orientation angle, and experimental results. (a) Energy map for a SWNT with radius $0.6 \mathrm{~nm}$ on quartz. The results show a single preferred orientation, at $0^{\circ}$. (b) SEM image of experimental results, showing behavior consistent with theory. Scale bar, $20 \mu \mathrm{m}$. The orange arrow indicates the flow direction. In a typical case such as this one, more than $99.91 \%$ of the total lengths of the SWNTs lie along the preferred direction, not including the regions where the catalyst particles are located.

roughness, the dependence on atomic arrangements at the surface is extremely strong, as might be expected based on the modeling results of parts $\mathrm{c}$ and $\mathrm{d}$ of Figure 2. With modeling, one can consider, for example, the case where the surface of the quartz is amorphous. Figure S9 in Supporting Information shows a calculated energy map on an amorphous quartz surface with an atomic structure generated by Materials Studio. ${ }^{49}$ Consistent with expectation, no single preferred direction appears in this case, similar to growth on thermal $\mathrm{SiO}_{2}$. More important, calculations show that a layer of amorphous quartz with thickness of only 0.1 $\mathrm{nm}$ on $\mathrm{Y}$ cut quartz reduces the energy well depth by $60 \%$. This result might explain reports of improved alignment on quartz due to annealing prior to the growth. ${ }^{50}$

Most previous experimental reports of aligned SWNTs on quartz used the widely available ST cut, ${ }^{15-23}$ which is a type of rotated Y cut that has a cut angle of $42^{\circ} 45^{\prime}$. Growth of SWNTs on such substrates yields aligned tubes with configurations nearly indistinguishable from those on the $\mathrm{Y}$ cut. Figure 5a shows the energy map of a SWNT of radius $0.6 \mathrm{~nm}$ on ST cut quartz, which predicts $0^{\circ}$ ( $x$ direction) to be the preferred growth direction. This outcome agrees with previously reported experiments. ${ }^{15-23}$ Figure 5 b shows an SEM image of SWNTs grown on ST cut quartz. No misaligned SWNTs occur within this field of view. In fact, with proper substrate annealing, patterned catalyst, and optimized growth conditions, it is routinely possible to achieve alignment yields, as defined by the percentage of 

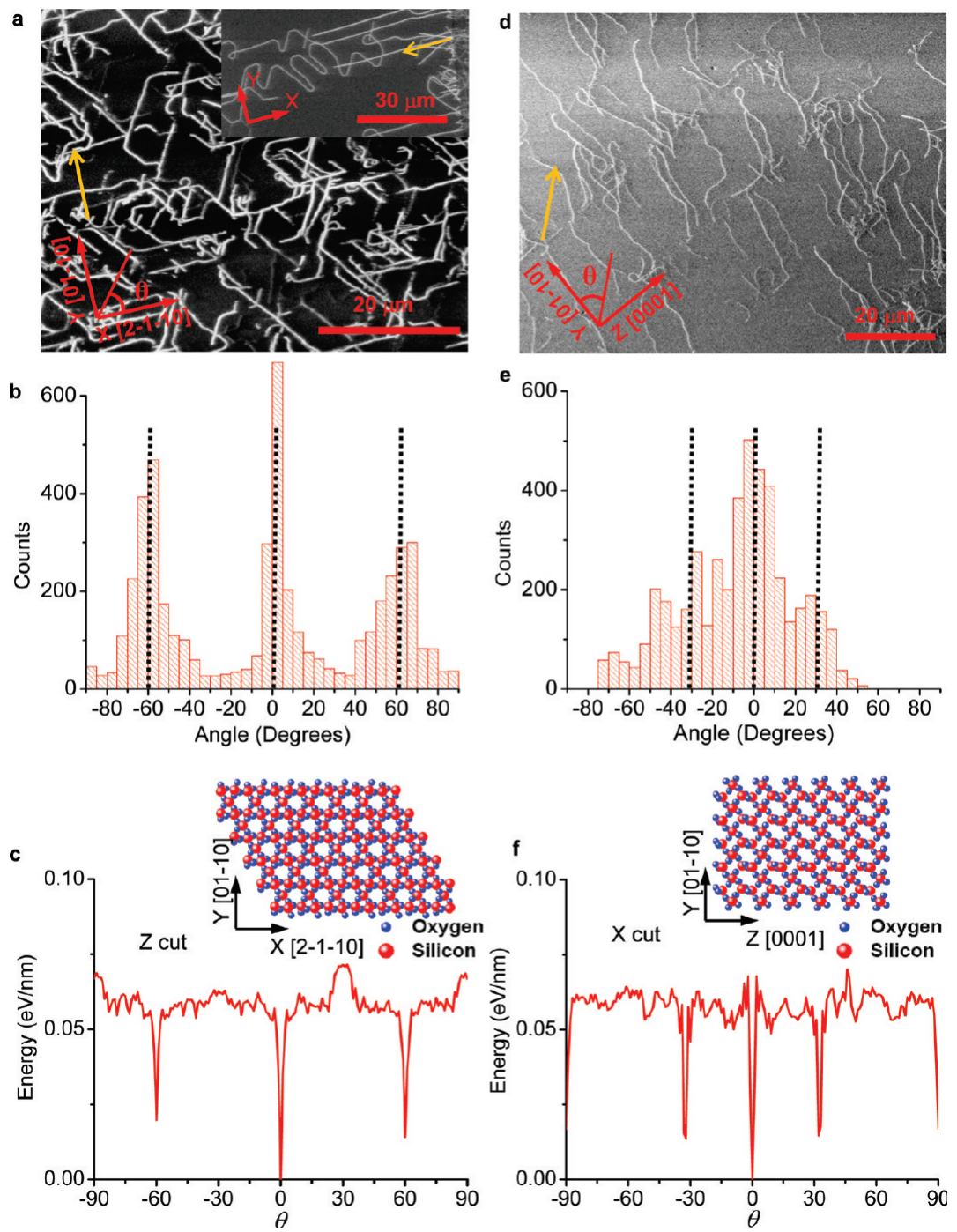

Figure 6. Experimental results for SWNTs grown on Z and X cut quartz, and corresponding calculations of the dependence of interaction energy on orientation angle. (a) SEM image of SWNTs grown on Z cut quartz. The inset shows a SWNT that grew above the quartz and then dropped to the surface after growth. The orange arrows indicate the gas flow direction. (b) Histogram of measured orientations of segments of SWNTs examined by AFM analysis. This procedure reveals three preferred orientation directions, each of which can be seen in (a). (c) Energy map for a SWNT with radius $0.6 \mathrm{~nm}$ on $\mathrm{Z}$ cut quartz. The predicted orientation angles are consistent with experiment. (d) SEM image of SWNTs grown on X cut quartz. The orange arrow indicates the gas flow direction. (e) Histogram of measured orientations of segments of SWNTs examined by AFM analysis. This procedure reveals a broad distribution of orientations, centered around $0^{\circ}$. Diffuse peaks at $\pm 30^{\circ}$ indicate a slight preference toward these directions. (f) Energy map for a SWNT with radius $0.6 \mathrm{~nm}$ on X cut quartz. The predicted orientation angles that span the range of orientations are dominate in the experimental results.

the total length of SWNTs that are aligned, of greater than $99.91 \%$, for regions away from the catalyst particles.

Growth on other cuts of quartz provides additional tests of the theory and also illustrates the degree to which substrate configuration can determine the layouts of the SWNTs. Figure 6a shows experimental results for $\mathrm{Z}$ cut quartz. Three directions are clearly prominent, as quantified with a histogram (Figure 6b) that shows the population of tube segments aligned in different directions. This plot was produced by dividing AFM images of tubes into a series of straight segments, each $150 \mathrm{~nm}$ long, and then recording their orientations. Figure $6 \mathrm{c}$ presents the energy map calculated for this case and the surface atomic structure in the inset. The results indicate minima along three directions: $0^{\circ}(x$ direction, $[2 \overline{1} \overline{1} 0]), 60^{\circ}([11 \overline{2} 0])$, and $-60^{\circ}([1 \overline{2} 10])$, consistent with the results of Figure $6 \mathrm{a}$.
The case of X cut substrates, shown in Figure 6d, provides a different type of result. Although the apparent alignment is poor compared to the $\mathrm{Y}$ and $\mathrm{Z}$ cuts, the SWNTs still exhibit some rough, overall orientation, in this case along the $y$ direction. A histogram analysis similar to that in Figure 6e summarizes the results. Figure of shows the computed energy map and surface atomic structure. This map indicates minima along four directions: $0^{\circ}(y$ direction, [01 $\overline{1} 0]$ ), $90^{\circ}$ ( $z$ direction, [0001]), and $32^{\circ}$ and $-32^{\circ}$, each with approximately the same energy well depth. We speculate that this situation allows the SWNTs to switch from the global minimum along $0^{\circ}$ ( $y$ direction) to the $\pm 32^{\circ}$ directions and that such switching can happen many times during the growth. Such a mechanism is consistent with the experimental results in Figure 6d. As with the Y cut quartz, the higher energy required to bend from $0^{\circ}$ might explain 
the relative absence of orientations along the minimum at $90^{\circ}$.

In summary, we have presented comprehensive theoretical and experimental studies of the process of alignment of SWNTs on quartz with various orientations. The collective body of results is consistent with an underlying physics governed by angle-dependent van der Waals interactions with the substrate. Other effects such as those related to surface polarization and piezoelectricity can contribute, but analysis suggests that their magnitudes are comparatively small. (See Supporting Information.) An intuitive picture therefore emerges in which the SWNTs orient along molecular-scale topological grooves that exist in certain directions on the surface of the substrate. The crystalline quality of the surface is paramount, while small features of surface relief or general surface roughness are relatively unimportant. This body of work might provide insights into formation of aligned configurations of SWNT on other crystalline substrates and, more generally, into strategies for nonepitaxial, orientation controlled growth of other classes of nanomaterials.

Methods. For all experiments SWNTs were grown by $\mathrm{CVD}$ at $925^{\circ} \mathrm{C}$ for $20 \mathrm{~min}$, with a flow of $20 \mathrm{sccms}$ of $\mathrm{Ar}$ and $20 \mathrm{sccms}$ of $\mathrm{H}_{2}$, bubbled through chilled $\left(0^{\circ} \mathrm{C}\right)$ ethanol. The growths were catalyzed by $\mathrm{Fe}$ nanoparticles dispersed on the substrate in one of two ways, both designed to minimize the interaction of unreacted catalyst particles with the SWNTs. In the first, a solution of ferritin (Sigma Aldrich) diluted 20000 times in deionized water was dispersed over the surface to form a bead of solution that covers the entire sample. Drops of methanol were then applied one at a time to each region of the substrate to cause particles to precipitate from the solution and coat the surface. Roughly $30 \mathrm{~s}$ was allowed between drops. Finally, the total volume of liquid was rinsed away with methanol and the sample was blown dry with $\mathrm{N}_{2}$. Heating to $850{ }^{\circ} \mathrm{C}$ for several minutes eliminated the organics, leaving $\mathrm{Fe}$ nanoparticles. While nanoparticle densities varied considerably from substrate to substrate, they tended to be $\sim 10-15$ nanoparticles/ $\mu \mathrm{m}$, based on AFM, for the results reported here. In the second approach, thin films of Fe $(0.1 \mathrm{~nm}$, nominal) were deposited (electron beam evaporation; Temescal) at a high angle $\left(\sim 80^{\circ}\right)$ onto $1-2$ $\mu \mathrm{m}$ (either lines or dot arrays) openings in photolithographically patterned layers of photoresist (Shipley S1805; 500 $\mathrm{nm})$. The photoresist was removed with a brief rinse in acetone. The resulting substrates were annealed at $950{ }^{\circ} \mathrm{C}$ for $1.5 \mathrm{~h}$ to form sparse coverages of iron nanoparticles in regions corresponding to those defined in the photoresist. In both approaches the samples were heated to the growth temperature in $300 \mathrm{sccms}$ of $\mathrm{H}_{2}$ to reduce the Fe.

SEM images were collected using an Hitachi S-4800 SEM at an operating voltage of $1 \mathrm{kV}$. AFM images were collected using an Asylum MFP3D AFM with Budgetsensor AFM tips. For all images, scan speeds were less than $20 \mu \mathrm{m} / \mathrm{s}$; for those used to define the histograms, the speeds were less than $10 \mu \mathrm{m} / \mathrm{s}$; for those used to determine diameters, speeds were less than $2.5 \mu \mathrm{m} / \mathrm{s}$.

All quartz wafers (Hoffman materials) were cleaned by rinsing with solvents (acetone, isopropyl alcohol, deionized water) and annealed for $8 \mathrm{~h}$ at $900{ }^{\circ} \mathrm{C}$. Y-cut wafers were polished using $0.1,0.25$, and $0.5 \mu \mathrm{m}$ grit diamond paper to remove step edges associated with manufacturer cleaning (5\% HF and saturated ammonium bifluoride). Various grits and anneal times $\left(900{ }^{\circ} \mathrm{C}\right)$ yielded samples with different levels of surface roughness.

Orientation histograms were generated by tracing SWNTs in SEM and AFM images using the NeuronJ application for ImageJ. This processing provided two-tone images and $x, y$ coordinates for the traced SWNTs. Line segments were created between points with a fixed distance from one another along the length of the SWNTs using MATLAB. There angles were recorded and used to generate histograms. The segments $(\sim 250 \mathrm{~nm})$ were selected to be as long as possible, without exceeding the characteristic length of straight regions of the SWNTs. (See Supporting Information for additional discussion.)

Acknowledgment. We thank T. Banks for help with processing and S. MacLaren for his help with AFM imaging, using facilities at the Frederick Seitz Materials Research Laboratory. This material is based upon work supported by a National Security Science and Engineering Faculty Fellowship and by the US Department of Energy, Division of Materials Sciences under Award No. DEFG02-07ER46453, through the Frederick Seitz MRL and Center for Microanalysis of Materials at the University of Illinois at Urbana-Champaign. S.D. acknowledges support from the Department of Defense (DOD) through the National Defense Science and Engineering Graduate (NDSEG) Fellowship program. Y.H. and K.-C.H. acknowledge support from the National Natural Science Foundation of China (NSFC).

Supporting Information Available: The interaction between SWNTs and quartz, the effect of polarization, the effect of quartz surface termination, the piezoelectric effect of quartz, energy maps of alpha and beta quartz, a SWNT climbing over a step, effect of step edges on SWNT alignment, orientation histograms, and effect of roughness on orientation. This material is available free of charge via the Internet at http://pubs.acs.org.

\section{References}

(1) Pesetski, A. A.; Baumgardner, J. E.; Krishnaswamy, S. V.; Zhang, H.; Adam, J. D.; Kocabas, C.; Banks, T.; Rogers, J. A. Appl. Phys. Lett. 2008, 93, 123506.

(2) Kocabas, C.; Kim, H.-S.; Banks, T.; Rogers, J. A.; Pesetski, A. A.; Baumgardner, J. E.; Krishnaswamy, S. V.; Zhang, H Proc. Natl Acad. Sci. U.S.A. 2008, 105, 1405-1409.

(3) Kocabas, C.; Dunham, S.; Cao, Q.; Cimino, K.; Ho, X.; Kim, H.-S.; Dawson, D.; Payne, J.; Stuenkel, M.; Zhang, H.; Banks, T.; Feng, M.; Rotkin, S. V.; Rogers, J. A. Nano Lett. 2009, 9, 1937-1943.

(4) Kang, S. J.; Kocabas, C.; Ozel, T.; Shim, M.; Pimparkar, N.; Alam, M. A.; Rotkin, S. V.; Rogers, J. A. Nat. Nanotechnol. 2007, 2, 230236.

(5) Cao, Q.; Kim, H.-S.; Pimparkar, N.; Kulkarni, J. P.; Wang, C.; Shim, M.; Roy, K.; Alam, M. A.; Rogers, J. A. Nature 2008, 454, 495-500.

(6) Ahn, J.-H.; Kim, H.-S.; Lee, K.-J.; Jeon, S.; Kang, S. J.; Sun, Y.; Nuzzo, R. G.; Rogers, J. A. Science 2006, 314, 1754-1757.

(7) Meitl, M. A.; Zhou, Y.; Gaur, A.; Jeon, S.; Usrey, M. L.; Strano, M. S.; Rogers, J. A. Nano Lett. 2004, 4, 1643-1647.

(8) Jin, Z.; Chu, H.; Wang, J.; Hong, J.; Tan, W.; Li, Y. Nano Lett. 2007, 7, 2073. 
(9) Zheng, L. X.; O’Connell, M. J.; Doorn, S. K.; Liao, X. Z.; Zhao, Y. H.; Akhadov, E. A.; Hoffbauer, M. A.; Roop, B. J.; Jia, Q. X.; Dye, R. C.; Peterson, D. E.; Huang, S. M.; Liu, J.; Zhu, Y. T. Nat. Mater. 2004, 3, 673-676.

(10) LeMieux, M. C.; Roberts, M.; Barman, S.; Jin, Y. W.; Kim, J. M.; Bao, Z. Science 2008, 321, 101-104.

(11) Krupke, R.; Hennrich, F.; Lohneysen, H. v.; Kappes, M. M. Science 2003, 301, 344-347.

(12) Zhang, Y. G.; Cao, J.; Wang, Q.; Kim, W.; Li, Y. M.; Morris, N.; Yenilmez, E.; Kong, J.; Dai, H. J. Appl. Phys. Lett. 2001, 79, 3155.

(13) Engel, M.; Small, J. P.; Steiner, M.; Freitag, M.; Green, A. A.; Hersam, M. C.; Avouris, P. ACS Nano 2008, 2, 2445-2452.

(14) Li, X.; Zhang, L.; Wang, X.; Shimoyama, I.; Sun, X.; Seo, W.-S.; Dai, H. J. Am. Chem. Soc. 2007, 129, 4890-4891.

(15) Kocabas, C.; Hur, S.-H.; Gaur, A.; Meitl, M. A.; Shim, M.; Rogers, J. A. Small 2005, 1, 1110-1116.

(16) Ding, L.; Yuan, D.; Liu, J. J. Am. Chem. Soc. 2008, 130, 5428-5429.

(17) Kocabas, C.; Kang, S. J.; Ozel, T.; Shim, M.; Rogers, J. A. J. Phys. Chem. C 2007, 111, 17879-17886.

(18) Zhou, W.; Rutherglen, C.; Burke, P. Nano Res. 2008, 1, 158.

(19) Ryu, K.; Badmaev, A.; Gomez, L.; Ishikawa, F.; Lei, B.; Zhou, C. J. Am. Chem. Soc. 2007, 129, 10104-10105.

(20) Ryu, K.; Badmaev, A.; Wang, C.; Lin, A.; Patil, N.; Gomez, J.; Kumar, A.; Mitra, S.; Wong, H. S.; Zhou, C. Nano Lett. 2009, 9, 189.

(21) Ding, L.; Tselev, A.; Wang, J.; Yuan, D.; Chu, H.; McNicholas, T.; Li, Y.; Liu, J. Nano Lett. 2009, 9, 800.

(22) Yuan, D.; Ding, D.; Chu, H.; Feng, Y.; McNicholas, T.; Liu, J. Nano Lett. 2008, 8, 2576.

(23) Ishikawa, F.; Chang, H.-K.; Ryu, K.; Chen, P.-C.; Badmaev, A.; Gomez, L.; Arco, D.; Shen, G.; Zhou, C. ACS Nano 2009, 3, 733.

(24) Koster Van Groos, A. F.; Ter Heege, J. P. J. Geol. 1973, 81, $717-$ 724.

(25) Ackermann, R. J.; Sorrell, C. A. J. Appl. Crystallogr. 1974, 7, 461467.

(26) Holmquist, S. B. J. Am. Ceram. Soc. 1961, 44, 82-86.

(27) Geblinger, N.; Ismach, A.; Joselevich, E. Nat. Nanotechnol. 2008, 3, 195-200.

(28) Jeon, S.; Lee, C.; Tang, J.; Hone, J.; Nuckolls, C. Nano Res. 2008, 1 427-433.

(29) Brenner, D. W.; Shenderova, O. A.; Harrison, J. A.; Stuart, S. J.; Ni, B.; Sinnott, S. B. J. Phys.: Condens. Matter 2002, 14, 783-802.
(30) Girifalco, L. A.; Hodak, M.; Lee, R. S. Phys. Rev. B 2000, 62, 13104 13110.

(31) van Duin, A. C. T.; Strachan, A.; Stewman, S.; Zhang, Q. S.; Xu, X.; Goddard, W. A. ReaxFF reactive force field for silicon and silicon oxide systems. J. Phys. Chem. A 2003, 107, 3803-3811.

(32) Bart, F.; Gautier, M.; Duraud, J. P.; Menriot, M. Surf. Sci. 1992, 274, 317-328.

(33) de Leeuw, N. H.; Manon Higgins, F.; Parker, S. C. J. Phys. Chem. B 1999, 103, 1270-1277.

(34) Rignanese, G.-M.; De Vita, A.; Charlier, J.-C.; Gonze, X.; Car, R. Phys. Rev. B 2000, 61, 13250-13255.

(35) Yang, J.; Wang, E. G. Phys. Rev. B 2006, 73, 035406.

(36) Goumans, T. P. M.; Wander, A.; Brown, W. A.; Catlow, C. R. A. Phys. Chem. Chem. Phys. 2007, 9, 2146-2152.

(37) Allen, M. P.; Tildesley, D. J. Computer Simulation of Liquids; Clarendon Press: Oxford, 1987.

(38) Zhang, L.; Jiang, S. J. Chem. Phys. 2002, 117, 1804-1811.

(39) Talu, O.; Myers, Colloids Surf., A 2001, 187-188, 83-93.

(40) Jiang, L. Y.; Huang, Y.; Jiang, H.; Ravichandran, G.; Gao, H.; Hwang, K. C.; Liu, B. J. Mech. Phys. Solids 2006, 54, 2436-2452.

(41) Tan, H.; Jiang, L. Y.; Huang, Y.; Liu, B.; Hwang, K. C. Compos. Sci. Technol. 2007, 67, 2941-2946.

(42) Israelachvili, J. N. Intermolecular and Surface Forces; Academic Press: London, 1992.

(43) Benedict, L. X.; Louie, S. G.; Cohen, M. L. Phys. Rev. B 1995, 52, 8541-8549.

(44) Massoud, H. Z. J. Appl. Phys. 1988, 63, 2000-2005.

(45) Nye, J. Physical Properties of Crystals; Clarendon Press: Oxford, 1957.

(46) Zhou, W.; Huang, Y.; Liu, B.; Wu, J.; Hwang, K. C.; Wei, B. Q. NANO 2007, 2, 175-179.

(47) Khang, D.-Y.; Xiao, J.; Kocabas, C.; MacLaren, S.; Banks, T.; Jiang, H.; Huang, Y. Y.; Rogers, J. A. Nano Lett. 2008, 8, 124-130.

(48) Xiao, J.; Jiang, H.; Khang, D.-Y.; Wu, J.; Huang, Y.; Rogers, J. A. J. Appl. Phys. 2008, 104, 033543.

(49) Materials Studio User's Manual Version 4.4; Accelrys Software Inc.: San Diego, CA, 2008.

(50) Pimparkar, N.; Kocabas, C.; Kang, S. J.; Rogers, J.; Alam, M. A. IEEE Electron Device Lett. 2007, 28, 593-595. (2007).

NL9025488 